\title{
On a set of transformations of Gaussian random functions
}

\author{
A.I. Nazarov1 \\ Dept. of Mathematics and Mechanics, \\ St.Petersburg State University \\ e-mail: an@AN4751.spb.edu
}

\begin{abstract}
We consider a set of one-dimensional transformations of Gaussian random functions. Under natural assumptions we obtain a connection between $L_{2}$-small ball asymptotics of the transformed function and of the original one. Also the explicit Karhunen - Loéve expansion is obtained for a proper class of Gaussian processes.
\end{abstract}

\section{Introduction and Main Lemma}

Recently P. Deheuvels [De] showed that for a standard Brownian bridge $B(t), 0 \leq t \leq 1$, the distributional equality

$$
\mathcal{Y}_{K}(t) \stackrel{d}{=} \mathcal{Y}_{2-K}(t), \quad t \in[0,1], K \in \mathbb{R},
$$

holds true (here $\mathcal{Y}_{K}(t)=B(t)-6 K t(1-t) \int_{0}^{1} B(s) d s$ ). Moreover, he obtained the explicit Karhunen - Loéve $(\mathrm{KL})$ expansion for the process $\mathcal{Y}_{1}(t)$.

We introduce one-parameter sets of transformations for zero mean-value Gaussian random functions. These transformations satisfy a relation generalizing (1.1). If the $L_{2}$-norm of original function is finite a.s. we derive the explicit relation between exact asymptotics of $L_{2}$-small ball probabilities for the transformed function and for the original one. For one-dimensional processes generating boundary value problems to ordinary differential equations we obtain also the KL-expansion for transformed process provided the KL-expansion for original process is known.

Let us consider a zero mean-value Gaussian random function $X(x), x \in \overline{\mathcal{O}}$, with the covariance $G_{X}(x, y)=\mathbb{E} X(x) X(y), x, y \in \overline{\mathcal{O}}$. For simplicity we suppose that $\mathcal{O}$ is a bounded domain in $\mathbb{R}^{n}$.

Let $\varphi$ be a locally summable function in $\mathcal{O}$. Suppose that the function

$$
\psi(x)=\int_{\mathcal{O}} G_{X}(x, y) \varphi(y) d y
$$

is well defined a.e. in $\mathcal{O}, \psi \neq \equiv 0$, and

$$
q=\int_{\mathcal{O}} \psi(u) \varphi(u) d u=\int_{\mathcal{O}} \int_{\mathcal{O}} G_{X}(u, v) \varphi(u) \varphi(v) d u d v<\infty .
$$

We define a set of Gaussian functions

$$
\mathcal{X}_{\varphi, \alpha}(x)=X(x)-\alpha \psi(x) \int_{\mathcal{O}} X(u) \varphi(u) d u, \quad x \in \overline{\mathcal{O}} .
$$

\footnotetext{
${ }^{1}$ Partially supported by grant NSh.227.2008.1 and by RFBR grant 07-01-00159.
} 
MAIN LEMMA. The covariance of $\mathcal{X}_{\varphi, \alpha}$ is

$$
\mathcal{G}_{\varphi, \alpha}(x, y)=G_{X}(x, y)+Q \psi(x) \psi(y),
$$

where $Q=q \alpha^{2}-2 \alpha$.

Proof. The formula (1.5) can be checked by direct computation due to (1.2).

Corollary 1. The equality

$$
\mathcal{X}_{\varphi, \alpha}(x) \stackrel{d}{=} \mathcal{X}_{\varphi, \frac{2}{q}-\alpha}(x), \quad x \in \overline{\mathcal{O}} .
$$

holds for the processes (1.4). In particular, $\mathcal{X}_{\varphi, \frac{2}{q}}(x) \stackrel{d}{=} X(x)$.

Corollary 2. Let $\widehat{\alpha}=\frac{1}{q}$. Then

1. The following identity holds true a.s.:

$$
\int_{\mathcal{O}} \mathcal{X}_{\varphi, \widehat{\alpha}}(x) \varphi(x) d x=0 .
$$

2. The process $\mathcal{X}_{\varphi, \widehat{\alpha}}(x)$ and the r.v. $\int_{\mathcal{O}} X(u) \varphi(u) d u$ are independent.

3. If $\varphi \in L_{2}(\mathcal{O})$ then the integral operator with the kernel function $\mathcal{G}_{\varphi, \widehat{\alpha}}(x, y)$ has a zero eigenvalue with the eigenfunction $\varphi$.

Proof. All three statements follow from relations

$$
\begin{gathered}
\int_{\mathcal{O}} \mathcal{X}_{\varphi, \alpha}(x) \varphi(x) d x=\int_{\mathcal{O}} X(u) \varphi(u) d u \cdot(1-q \alpha) ; \\
\mathbb{E} \mathcal{X}_{\varphi, \alpha}(x) \int_{\mathcal{O}} X(u) \varphi(u) d u=\psi(x) \cdot(1-q \alpha),
\end{gathered}
$$

which can be easily checked.

Remark. Trivially the process $\mathcal{Y}_{K}$ coincides with $\mathcal{B}_{\varphi, \alpha}$ for $\varphi \equiv 1, \alpha=12 K$. Hence, Lemma 2.2 and Corollaries 2.1, 2.2 from [De] are particular cases of our statements.

\section{The asymptotics of small ball probabilities in $L_{2}$}

Now we suppose that

$$
\|X\|_{2}^{2} \equiv \int_{\mathcal{O}} X^{2}(x) d x<\infty \quad \text { a.s. }
$$

Then the process $X$ admits the KL-expansion

$$
X(x) \stackrel{d}{=} \sum_{k=1}^{\infty} \sqrt{\lambda_{k}} u_{k}(x) \xi_{k}, \quad x \in \mathcal{O},
$$

where $\xi_{k}$ is a sequence of independent standard Gaussian r.v. while $\lambda_{k}>0$ and $u_{k}$ are, respectively, eigenvalues and (normalized in $L_{2}(\mathcal{O})$ ) eigenfunctions of the integral operator $\mathfrak{G}$ 
with the kernel function $G_{X}(x, y)$. Moreover, (2.1) implies $\sum_{k} \lambda_{k}<\infty$ i.e the operator $\mathfrak{G}$ belongs to the kernel class $\mathfrak{S}_{1}$. Remark that the series $(2.2)$ converges in $L_{2}(\mathcal{O})$ a.s.

The relation (2.2) implies

$$
\|X\|_{2}^{2} \stackrel{d}{=} \sum_{k=1}^{\infty} \lambda_{k} \xi_{k}^{2}
$$

Therefore, having the eigenvalues $\lambda_{k}$ in hands one can obtain some information on the distribution of $\|X\|_{2}^{2}$. In particular, one can derive the exact asymptotics of small ball probabilities in $L_{2}$ i.e. describe the behavior of the probability $\mathbb{P}\left\{\|X\|_{2} \leq \varepsilon\right\}$ as $\varepsilon \rightarrow 0$.

In [Syt, a solution of the small ball behavior problem was obtained in abstract form. Then many authors simplified formulas for small ball probabilities under various assumptions; see [DLL] and references therein.

In papers [NN], Na], [Na1] a new approach was delivered. This approach provides the exact $L_{2}$-small ball asymptotics for a zero mean-value Gaussian process if its covariance is the Green function for an ordinary differential operator. In more general case the problem cannot be solved completely yet. However, in the case under consideration the transformed operator is a one-dimensional perturbation of the original one. So, we can derive the small ball asymptotics for the process $\mathcal{X}_{\varphi, \alpha}$ in terms of the small ball asymptotics for the original process.

Remark. If the operator $\mathfrak{G}$ has a nontrivial null-space $U_{0}$ then it is contained in the null-space of the operator with the kernel function $\mathcal{G}_{\varphi, \alpha}(x, y)$ due to obvious relation $\psi \perp U_{0}$. Therefore, we can apply all the arguments in an orthogonal complement to $U_{0}$ (i.e. in the image of $\mathfrak{G})$. So, without loss of generality one can assume $\left\{u_{k}\right\}$ in (2.2) being an orthogonal basis.

Theorem 1. Let the process $X$ satisfy (2.1). Suppose that a function $\varphi \in L_{1, l o c}(\mathcal{O})$ satisfies (1.3). If $\alpha \neq \frac{1}{q}$ then, as $\varepsilon \rightarrow 0$,

$$
\mathbb{P}\left\{\left\|\mathcal{X}_{\varphi, \alpha}\right\|_{2} \leq \varepsilon\right\} \sim \frac{1}{|1-\alpha q|} \cdot \mathbb{P}\left\{\|X\|_{2} \leq \varepsilon\right\}
$$

Proof. By comparison theorem ([Li]; see also [GHT]),

$$
\mathbb{P}\left\{\left\|\mathcal{X}_{\varphi, \alpha}\right\|_{2} \leq \varepsilon\right\} \sim \mathbb{P}\left\{\|X\|_{2} \leq \varepsilon\right\} \cdot\left(\prod_{k=1}^{\infty} \frac{\lambda_{k}}{\widetilde{\lambda}_{k}}\right)^{1 / 2}
$$

where $\widetilde{\lambda}_{k}$ are the eigenvalues of the integral operator with kernel function $\mathcal{G}_{\varphi, \alpha}(x, y)$. Remark that due to the minimax principle, see, e.g., [BS, §10.2]) the sequences $\lambda_{k}$ and $\widetilde{\lambda}_{k}$ interlace. In particular, this implies the convergence of the series $\sum_{k} \widetilde{\lambda}_{k}$.

By definition, put $\mu_{k}=\lambda_{k}^{-1}, \widetilde{\mu}_{k}=\widetilde{\lambda}_{k}^{-1}$. Consider the Fredholm determinants for the kernels $G_{X}$ and $\mathcal{G}_{\varphi, \alpha}$ :

$$
\mathcal{F}(z)=\prod_{k=1}^{\infty}\left(1-\frac{z}{\mu_{k}}\right) ; \quad \widetilde{\mathcal{F}}(z)=\prod_{k=1}^{\infty}\left(1-\frac{z}{\widetilde{\mu}_{k}}\right) .
$$

Since the series $\sum_{k} \mu_{k}^{-1}$ and $\sum_{k} \widetilde{\mu}_{k}^{-1}$ converge, these canonical Hadamard's products converge for 
all $z \in \mathbb{C}$. By (1.5) the following relation holds true?:

$$
\widetilde{\mathcal{F}}(z)=\mathcal{F}(z) \cdot\left(1+Q \sum_{k=1}^{\infty} \frac{a_{k}^{2} \mu_{k}}{1-\frac{\mu_{k}}{z}}\right)
$$

where $a_{k}$ are the Fourier coefficients of the function $\psi$ with respect to the system $\left\{u_{k}\right\}$.

The Jensen theorem, see [Ti, §3.6], provides

$$
\prod_{k=1}^{\infty} \frac{\mu_{k}}{\widetilde{\mu}_{k}}=\lim _{|z| \rightarrow \infty} \exp \left(\frac{1}{2 \pi} \int_{0}^{2 \pi} \ln \left(\frac{|\widetilde{\mathcal{F}}(z)|}{|\mathcal{F}(z)|}\right) d \arg (z)\right) .
$$

Formula (2.5) and Lemma 5.1 show that the last limit equals $\left|1+Q \sum_{k} a_{k}^{2} \mu_{k}\right|$. But $\psi=\sum_{k} a_{k} u_{k}$ implies $\varphi=\sum_{k} \mu_{k} a_{k} u_{k}$ and, therefore,

$$
\sum_{k=1}^{\infty} a_{k}^{2} \mu_{k}=\int_{\mathcal{O}} \psi(u) \varphi(u) d u=q
$$

Substituting (2.6) into (2.4) we obtain (2.3).

Now we consider the critical case $\widehat{\alpha}=\frac{1}{q}$.

Theorem 2. Let the process $X$ satisfy (2.1), and let $\widehat{\alpha}=\frac{1}{q}$. If $\varphi \in L_{2}(\mathcal{O})$ then, as $\varepsilon \rightarrow 0$,

$$
\mathbb{P}\left\{\left\|\mathcal{X}_{\varphi, \widehat{\alpha}}\right\|_{2} \leq \varepsilon\right\} \sim \frac{\sqrt{q}}{\|\varphi\|_{2}} \cdot \sqrt{\frac{2}{\pi}} \cdot \int_{0}^{\varepsilon^{2}} \frac{d}{d t} \mathbb{P}\left\{\|X\|_{2} \leq t\right\} \frac{d t}{\sqrt{\varepsilon^{2}-t^{2}}}
$$

Proof. We introduce three distribution functions:

$$
\begin{aligned}
F(r) & =\mathbb{P}\left\{\sum_{k=1}^{\infty} \lambda_{k} \xi_{k}^{2} \leq r\right\}=\mathbb{P}\left\{\|X\|_{2} \leq \sqrt{r}\right\} ; \\
\widetilde{F}(r) & =\mathbb{P}\left\{\sum_{k=1}^{\infty} \widetilde{\lambda}_{k} \xi_{k}^{2} \leq r\right\}=\mathbb{P}\left\{\left\|\mathcal{X}_{\varphi, \widehat{\alpha}}\right\|_{2} \leq \sqrt{r}\right\} ; \\
F_{1}(r) & =\mathbb{P}\left\{\sum_{k=2}^{\infty} \lambda_{k} \xi_{k}^{2} \leq r\right\} .
\end{aligned}
$$

Similarly to the previous theorem we have, as $r \rightarrow 0$,

$$
\widetilde{F}(r) \sim F_{1}(r) \cdot\left(\prod_{k=2}^{\infty} \frac{\widetilde{\mu}_{k}}{\mu_{k-1}}\right)^{1 / 2}
$$

\footnotetext{
${ }^{2}$ Note that (2.5) is a particular case of the transformation formula for the Fredholm determinant under finite-dimensional perturbation of the operator. In the literature on statistics this formula usually is attributed to $[\mathrm{Su}$. Statistical applications in $[\mathrm{Su}]$ seem to be new while the formula itself was really obtained in $\mathrm{Ba}$ and was well known as in computational methods as in spectral theory, even in more general situation, see, e.g., [KK, Ch.II, 4.6] and [AG, Sec.106].
} 
The Jensen theorem provides

$$
\prod_{k=2}^{\infty} \frac{\mu_{k-1}}{\widetilde{\mu}_{k}}=\lim _{|z| \rightarrow \infty} \exp \left(\frac{1}{2 \pi} \int_{0}^{2 \pi} \ln \left|\left(1-\frac{z}{\mu_{1}}\right) \cdot \frac{\tilde{\mathcal{F}}(z)}{\mathcal{F}(z)}\right| d \arg (z)\right) .
$$

The assumption $\widehat{\alpha}=\frac{1}{q}$ implies $Q=-\frac{1}{q}$. Hence, due to (2.5) and (2.7) the expression under log sign can be rewritten as follows:

$$
\left|\left(1-\frac{z}{\mu_{1}}\right) \cdot\left(1+Q \sum_{k=1}^{\infty} \frac{a_{k}^{2} \mu_{k}}{1-\frac{\mu_{k}}{z}}\right)\right|=\left|\frac{\frac{1}{\mu_{1}}-\frac{1}{z}}{q} \cdot \sum_{k=1}^{\infty} \frac{a_{k}^{2} \mu_{k}^{2}}{1-\frac{\mu_{k}}{z}}\right| .
$$

By Lemma 5.1, the limit in (2.9) equals

$$
\frac{1}{\mu_{1} q} \sum_{k=1}^{\infty} a_{k}^{2} \mu_{k}^{2}=\frac{\|\varphi\|_{2}^{2}}{\mu_{1} q}
$$

This gives

$$
\widetilde{F}(r) \sim F_{1}(r) \cdot \frac{\sqrt{q \mu_{1}}}{\|\varphi\|_{2}}
$$

Further, obviously, $F(r)=\left(F_{1} * f\right)(r)$, where

$$
f(x)=\frac{d}{d x} \mathbb{P}\left\{\lambda_{1} \xi^{2} \leq x\right\}=\frac{\exp \left(-\frac{x}{2 \lambda_{1}}\right)}{\sqrt{2 \pi \lambda_{1} x}} .
$$

By the Laplace transform we obtain a solution of this convolution equation:

$$
F_{1}(r)=\sqrt{\frac{2 \lambda_{1}}{\pi}} \exp \left(-\frac{r}{2 \lambda_{1}}\right) \int_{0}^{r}\left(F(x) \exp \left(\frac{x}{2 \lambda_{1}}\right)\right)^{\prime} \frac{d x}{\sqrt{r-x}} .
$$

Lemma 2.1. $F(x)=o\left(F^{\prime}(x)\right)$ as $x \rightarrow+0$.

Proof. Remark that $F^{\prime}$ is absolutely continuous on $\mathbb{R}$, if the sum (2.2) contains at least three nonzero summands. We claim that $F^{\prime \prime}>0$ in a right half-neighborhood of the origin. Indeed, this property can be directly checked for three summands and is easily conserved under adding a new summand; moreover, the radius of a neighborhood is not decreasing. Consequently, this property is conserved also for the infinite sum.

Since $F$ is convex in a right half-neighborhood of the origin, $F^{\prime}(x) \geq F(x) / x$ in this halfneighborhood.

We continue the proof of Theorem 2. By Lemma 2.1, we obtain from (2.11)

$$
F_{1}(r) \sim \sqrt{\frac{2 \lambda_{1}}{\pi}} \int_{0}^{r} F^{\prime}(x) \cdot \frac{\exp \left(-\frac{r-x}{2 \lambda_{1}}\right) d x}{\sqrt{r-x}} \sim \sqrt{\frac{2 \lambda_{1}}{\pi}} \int_{0}^{r} F^{\prime}(x) \frac{d x}{\sqrt{r-x}} .
$$

Substituting (2.12) into (2.10) and changing the variable $r=\varepsilon^{2}$ we arrive at (2.8).

Remark. By misuse of language, we can interpret (2.10) as follows. For $\alpha \neq \frac{1}{q}$ the sequences $\mu_{k}$ and $\widetilde{\mu}_{k}$ have the same asymptotics. Under the conditions of Theorem 2 , the statement 3 of Corollary 2 provides vanishing of an eigenvalue $\widetilde{\lambda}_{k}$. This generates a confusion in the enumeration of $\widetilde{\mu}_{k}$; removing of a summand from (2.2) we reestablish the corresponding of enumerations. 


\section{Karhunen - Loéve expansion}

Now we suppose that $n=1, \mathcal{O}=(0, a)$ is an interval, and the covariance $G_{X}(t, s), t, s \in[0, a]$, is the Green function of a self-adjoint operator $L_{X}$ in the space $L_{2}(0, a)$, generated by differential expression of order $2 \ell$

$$
L_{X} u \equiv(-1)^{\ell} u^{(2 \ell)}+\left(p_{\ell-1} u^{(\ell-1)}\right)^{(\ell-1)}+\cdots+p_{0} u
$$

and $2 \ell$ boundary conditions. We recall that by definition $G_{X}$ for any $s \in(0, a)$ satisfies the equation $L_{X} G_{X}=\delta(t-s)$ in the sense of distributions, and satisfies boundary conditions. Without loss of generality we assume $a=1$.

By $\mathcal{D}\left(L_{X}\right)$ denote the image of integral operator with the kernel function $G_{X}(t, s)$. Then it is easy to see that the inverse operator is just $L_{X}$ with the domain $\mathcal{D}\left(L_{X}\right)$. In particular, if $\varphi \in L_{2}(0,1)$ then $\psi \in \mathcal{D}\left(L_{X}\right)$, and $L_{X} \psi=\varphi$.

Assume for simplicity that $p_{j} \in \mathcal{C}^{j}[0,1]$. Then $\mathcal{D}\left(L_{X}\right)$ coincides with the set of functions which belong to $W_{2}^{\ell}(0,1)$ and satisfy boundary conditions. By $(1.5)$ we obtain that the covariance of transformed process $\mathcal{X}_{\varphi, \alpha}$ satisfies the equation

$$
L_{X} \mathcal{G}_{\varphi, \alpha}=\delta(t-s)+Q \varphi(t) \psi(s)
$$

(recall that $Q=q \alpha^{2}-2 \alpha$ ) and satisfies the same boundary conditions.

Suppose we know KL-expansion (2.2) for the original process. Then, obviously, $u_{k}$ are the eigenfunctions and $\mu_{k}=\lambda_{k}^{-1}$ are the eigenvalues of the boundary value problem

$$
L_{X} u=\mu u, \quad u \in \mathcal{D}\left(L_{X}\right) .
$$

In practice, the eigenfunctions of the problem (3.3) can be found analytically only if we know the fundamental system of solutions to the equation $L_{X} v-\mu v=0$ for arbitrary $\mu \in \mathbb{R}$. We take advantage of this to give an algorithm of derivation of KL-expansion for the process $\mathcal{X}_{\varphi, \alpha}$. Note that in a particular case this algorithm was used in [KKW] (see below the example 5).

From (3.2) we obtain the boundary value problem for eigenfunctions of integral operator with the kernel function $\mathcal{G}_{\varphi, \alpha}(t, s)$ :

$$
L_{X} u=\mu u+\mu Q \varphi \int_{0}^{1} u(s) \psi(s) d s, \quad u \in \mathcal{D}\left(L_{X}\right)
$$

Let $\mu$ be an unknown parameter. By the Lagrange method we can construct a particular solution to the equation $L_{X} \eta-\mu \eta=\varphi$. Then a general solution to the equation (3.4) can be written as follows:

$$
u=c_{0} \eta+c_{1} v_{1}+c_{2} v_{2}+\cdots+c_{2 \ell} v_{2 \ell},
$$

where $v_{1}, \ldots, v_{2 \ell}$ form a fundamental system for the equation $L_{X} v-\mu v=0$. Substituting $u$ into the boundary conditions we obtain $2 \ell$ equations for the constants $c_{0}, c_{1}, \ldots, c_{2 \ell}$. One more equation follows from the equality of the coefficients at $\varphi$ in (3.4):

$$
\frac{c_{0}}{\mu Q}=c_{0} \int_{0}^{1} \eta(s) \psi(s) d s+c_{1} \int_{0}^{1} v_{1}(s) \psi(s) d s+\cdots+c_{2 \ell} \int_{0}^{1} v_{2 \ell}(s) \psi(s) d s .
$$


Eigenvalues of the problem (3.4) are roots of the determinant of the obtained homogenous system while eigenfunctions are its nontrivial solutions.

Let us show some examples.

Example 1. Let $X=W$ be a standard Wiener process, and let $\varphi \equiv 1$. Then

$$
\psi(s)=\int_{0}^{1} \min (t, s) d s=\frac{2 t-t^{2}}{2} ; \quad q=\int_{0}^{1} \frac{2 t-t^{2}}{2} d t=\frac{1}{3} .
$$

The relation (3.4) reads as follows:

$$
-u^{\prime \prime}=\mu u+\frac{\mu Q}{2} \int_{0}^{1} u(s)\left(2 s-s^{2}\right) d s, \quad u(0)=u^{\prime}(1)=0 .
$$

A general solution of this equation is

$$
u(t)=c_{0}+c_{1} \cos (\omega t)+c_{2} \sin (\omega t), \quad \omega=\mu^{1 / 2} .
$$

Substituting it into the boundary conditions and into (3.5) we derive the equation for eigenvalues:

$$
Q \sin (\omega)=\cos (\omega) \cdot\left(Q \omega+(1+Q / 3) \omega^{3}\right) .
$$

For $Q=0$ we obtain $\cos (\omega)=0$. This is natural result because in this case we have a conventional Wiener process.

We remark also that $1+Q / 3=(1-\alpha / 3)^{2} \geq 0$. For $\alpha=3$ the equation (3.7) is reduced to $\tan (\omega)=\omega$.

Let $\omega_{k}$ be positive roots of (3.7) enumerated in the increasing order. Then we put in KLexpansion of the process $\mathcal{W}_{\mathbf{1}, \alpha}$

$$
\widetilde{\lambda}_{k}=\omega_{k}^{-2}, \quad \widetilde{u}_{k}(t)=\gamma_{k}\left(\cos \left(\omega_{k} t\right)-1+\tan \left(\omega_{k}\right) \sin \left(\omega_{k} t\right)\right),
$$

where $\gamma_{k}$ are the normalizing constants.

Example 2. Let $X=B$ be a standard Brownian bridge, and let $\varphi \equiv 1$. Then $\psi(t)=\frac{t-t^{2}}{2}$, $q=\frac{1}{12}$. The relation (3.4) reads as follows:

$$
-u^{\prime \prime}=\mu u+\frac{\mu Q}{2} \int_{0}^{1} u(s)\left(s-s^{2}\right) d s, \quad u(0)=u(1)=0 .
$$

A general solution of this equation is given by (3.6). Substituting it into the boundary conditions and into (3.5) we derive the equation for eigenvalues:

$$
\left[\begin{array}{rl}
\sin (\tau) & =0 \\
Q \sin (\tau) & =\cos (\tau) \cdot\left(Q \tau+(4+Q / 3) \tau^{3}\right), \quad \tau=\frac{\omega}{2}
\end{array}\right.
$$

For $Q=0$ two equations in (3.9) can be merged into $\sin (\omega)=0$, as is expected. We remark also that $4+Q / 3=(2-\alpha / 6)^{2} \geq 0$. For $\alpha=12$ the second equation in (3.9) is reduced to $\tan (\tau)=\tau$. This case was considered in [De, Theorem 1.2]. 
A sequence

$$
Q_{k}=-\frac{12}{1+\frac{3}{(k \pi)^{2}}} \searrow-12
$$

(any $Q_{k}$ corresponds to two values of $\alpha$ ) has a curious property. Namely, for $Q=Q_{k}$ the $k$-th roots of both equations in (3.9) coincide. Thus, for $Q=Q_{1}$ the least eigenvalue of the problem (3.8) is multiple. This effect is impossible for a conventional Sturm - Liouville problem.

Let $\tau_{k}$ be positive roots of the second equation in (3.9) enumerated in the increasing order. Then we put in KL-expansion of the process $\mathcal{B}_{1, \alpha}$

$$
\begin{array}{ll}
\widetilde{\lambda}_{k}=(2 k \pi)^{-2}, & \widetilde{u}_{k}(t)=\sqrt{2} \sin (2 \pi k t) ; \\
\widetilde{\widetilde{\lambda}}_{k}=\left(2 \tau_{k}\right)^{-2}, & \widetilde{\widetilde{u}}_{k}(t)=\gamma_{k}\left(\cos \left(2 \tau_{k} t\right)-1+\tan \left(\tau_{k}\right) \sin \left(2 \tau_{k} t\right)\right),
\end{array}
$$

where $\gamma_{k}$ are the normalizing constants.

Example 3. Let $X=B$, and let $\varphi(t)=t(1-t)$. Then $\psi(t)=\frac{t-2 t^{3}+t^{4}}{12}, q=\frac{17}{5040}$. The relation (3.4) reads as follows:

$$
-u^{\prime \prime}=\mu u+\frac{\mu Q}{12} t(1-t) \int_{0}^{1} u(s)\left(s-2 s^{3}+s^{4}\right) d s, \quad u(0)=u(1)=0 .
$$

A general solution of this equation is

$$
u(t)=c_{0}\left(t-t^{2}+\frac{2}{\omega^{2}}\right)+c_{1} \cos (\omega t)+c_{2} \sin (\omega t), \quad \omega=\mu^{1 / 2} .
$$

Substituting it into the boundary conditions and into (3.5) we derive the equation for eigenvalues:

$$
\left[\begin{array}{rl}
\sin (\tau) & =0 ; \\
Q \sin (\tau) & =\cos (\tau) \cdot\left(Q \tau+Q \tau^{3} / 3+2 Q \tau^{5} / 15+16(1+Q q) \tau^{7}\right), \quad \tau=\frac{\omega}{2}
\end{array}\right.
$$

For $Q=0$ two equations in $(3.10)$ can be merged into $\sin (\omega)=0$. We remark also that $1+Q q=(1-q \alpha)^{2} \geq 0$.

Let $\tau_{k}$ be positive roots of the second equation in (3.10) enumerated in the increasing order. Then we put in KL-expansion of the process $\mathcal{B}_{\varphi, \alpha}$

$$
\begin{array}{ll}
\widetilde{\lambda}_{k}=(2 k \pi)^{-2}, & \widetilde{u}_{k}(t)=\sqrt{2} \sin (2 \pi k t) ; \\
\widetilde{\vec{\lambda}}_{k}=\left(2 \tau_{k}\right)^{-2}, & \widetilde{\widetilde{u}}_{k}(t)=\gamma_{k}\left(\cos \left(2 \tau_{k} t\right)-1+2 \tau_{k}^{2}\left(t^{2}-t\right)+\tan \left(\tau_{k}\right) \sin \left(2 \tau_{k} t\right)\right),
\end{array}
$$

where $\gamma_{k}$ are the normalizing constants.

Example 4. Let

$$
X(t)=\bar{W}_{1}(t)=\int_{0}^{t}\left(W(s)-\int_{0}^{1} W(u) d u\right) d s
$$

be an integrated centered Wiener process. Its covariation $G_{\bar{W}_{1}}$ is the Green function of the operator $L_{\bar{W}_{1}}=L_{B}^{2}$, see, e.g., [HN] and [NN, Prop. 5.4]. 
If $\varphi \equiv 1$, then $\psi(t)=\frac{t-2 t^{3}+t^{4}}{24}, q=\frac{1}{120}$, and the relation (3.4) reads as follows:

$$
u^{I V}=\mu u+\frac{\mu Q}{24} \int_{0}^{1} u(s)\left(s-2 s^{3}+s^{4}\right) d s, \quad u(0)=u(1)=u^{\prime \prime}(0)=u^{\prime \prime}(1)=0 .
$$

A general solution of this equation is

$$
u(t)=c_{0}+c_{1} \cos (\omega t)+c_{2} \sin (\omega t)+c_{3} \cosh (\omega t)+c_{4} \sinh (\omega t), \quad \omega=\mu^{1 / 4} .
$$

Substituting it into the boundary conditions and into (3.5) we derive the equation for eigenvalues:

$$
\begin{aligned}
\sin (\tau) & =0 \\
Q(\sin (\tau)+\cos (\tau) \tanh (\tau)) & =\cos (\tau) \cdot\left(2 Q \tau+(32+4 Q / 15) \tau^{5}\right), \quad \tau=\frac{\omega}{2}
\end{aligned}
$$

For $Q=0$ two equations in (3.10) can be merged into $\sin (\omega)=0$. We remark also that $32+4 Q / 15=32(1-q \alpha)^{2} \geq 0$.

Let $\tau_{k}$ be positive roots of the second equation in (3.11) enumerated in the increasing order. Then we put in KL-expansion of the process $\mathcal{X}_{\mathbf{1}, \alpha}$

$$
\begin{aligned}
& \widetilde{\tilde{\lambda}}_{k}=(2 k \pi)^{-4}, \quad \widetilde{u}_{k}(t)=\sqrt{2} \sin (2 \pi k t) ; \\
& \tilde{\widetilde{\lambda}}_{k}=\left(2 \tau_{k}\right)^{-4}, \quad \widetilde{\widetilde{u}}_{k}(t)=\gamma_{k}\left(\cos \left(2 \tau_{k} t\right)+\cosh \left(2 \tau_{k} t\right)-2+\right. \\
& \left.+\tan \left(\tau_{k}\right) \sin \left(2 \tau_{k} t\right)-\tanh \left(\tau_{k}\right) \sinh \left(2 \tau_{k} t\right)\right) \text {, }
\end{aligned}
$$

where $\gamma_{k}$ are the normalizing constants.

It should be noted that the roots of the first equation in (3.9)-(3.11) are independent of $Q$. The reason is that corresponding eigenfunctions $\sin (2 \pi k t), k \in \mathbb{N}$, are orthogonal to $\psi$ in $L_{2}(0,1)$, and the last term in (3.4) vanishes.

Example 5. Let $X=B$, and let

$$
\varphi(t)=\frac{1}{\phi\left(\boldsymbol{\Phi}^{-1}(t)\right)}, \quad \phi(t)=\frac{1}{\sqrt{2 \pi}} \exp \left(-t^{2} / 2\right) ; \quad \boldsymbol{\Phi}(x)=\int_{-\infty}^{x} \phi(t) d t .
$$

This example is important in statistics, see [KKW], $\left[\mathrm{Su}\right.$. It is evident that $\varphi \notin L_{2}(0,1)$. However, the direct calculation gives $\psi=\phi\left(\boldsymbol{\Phi}^{-1}\right)=\frac{1}{\varphi}, q=1$. Hence all the statements of $\S 1$ are applicable here.

Further, the relation (3.4) reads as follows:

$$
-u^{\prime \prime}=\mu u+\frac{\mu Q}{\phi\left(\boldsymbol{\Phi}^{-1}(t)\right)} \int_{0}^{1} u(s) \phi\left(\boldsymbol{\Phi}^{-1}(s)\right) d s, \quad u(0)=u(1)=0 .
$$

A general solution of this equation is

$$
u(t)=c_{0} \int_{\frac{1}{2}}^{t} \frac{\sin (\omega(\tau-t)) d \tau}{\omega \phi\left(\boldsymbol{\Phi}^{-1}(\tau)\right)}+c_{1} \cos (\omega t)+c_{2} \sin (\omega t), \quad \omega=\mu^{1 / 2} .
$$


Substituting it into the boundary conditions and into (3.5) after some calculations we derive the equation for eigenvalues?:

$$
\operatorname{det}\left[\begin{array}{ccc}
0 & \int_{0}^{1} \boldsymbol{\Phi}^{-1}(\tau) \cos (\omega \tau) d \tau & -\frac{1}{\omega^{2} Q}+\int_{0}^{1} \int_{0}^{\tau} \boldsymbol{\Phi}^{-1}(\tau) \boldsymbol{\Phi}^{-1}(t) \frac{\sin (\omega(t-\tau))}{\omega} d t d \tau \\
\sin (\omega) & 0 & -\frac{1}{\omega} \int_{0}^{1} \boldsymbol{\Phi}^{-1}(\tau) \cos (\omega \tau) d \tau \\
\cos (\omega) & 1 & \frac{1}{\omega} \int_{0}^{1} \boldsymbol{\Phi}^{-1}(\tau) \sin (\omega \tau) d \tau
\end{array}\right]=0 .
$$

For $Q=0$ passing to the limit gives a natural result $\sin (\omega)=0$. We note that in this example also a half of eigenfunctions for the original process (namely, $\sin (2 \pi k t), k \in \mathbb{N}$ ) are orthogonal to $\psi$ in $L_{2}(0,1)$. Therefore, they do not depend on $Q$.

Now we describe a case where the KL-expansion for the transformed process can be constructed trivially. Let $\varphi=u_{m}$ be an eigenfunction of the covariance $G_{X}$. Then $\psi=\lambda_{m} u_{m}$, where $\lambda_{m}$ is corresponding eigenvalue. Hence all the eigenfunctions $u_{k}, k \neq m$, are orthogonal to $\psi$, and therefore,

$$
\begin{array}{ll}
\widetilde{\lambda}_{k}=\lambda_{k}, & \widetilde{u}_{k}=u_{k}, \quad k \neq m, \\
\widetilde{\lambda}_{m}=\lambda_{m}(1-q \alpha)^{2}, & \widetilde{u}_{m}=u_{m} .
\end{array}
$$

Now we establish the relation which simplifies (2.8) for the processes under consideration.

Theorem 3. Let the covariance $G_{X}$ be the Green function for an operator of the form (3.1), and let $\widehat{\alpha}=\frac{1}{q}$. If $\varphi \in L_{2}(0,1)$ then, as $\varepsilon \rightarrow 0$,

$$
\mathbb{P}\left\{\left\|\mathcal{X}_{\varphi, \widehat{\alpha}}\right\| \leq \varepsilon\right\} \sim \frac{\sqrt{q}}{\|\varphi\|_{2}} \cdot\left(2 \ell \sin \left(\frac{\pi}{2 \ell}\right) \varepsilon^{2}\right)^{-\frac{\ell}{2 \ell-1}} \cdot \mathbb{P}\{\|X\| \leq \varepsilon\} .
$$

Proof. It is shown in [Na1, Theorem 1.2] that the process $X$ satisfies the relation 4

$$
F(r)=\mathbb{P}\{\|X\| \leq \sqrt{r}\} \sim \mathcal{C} \cdot r^{\beta} \exp \left(-\mathfrak{D} r^{-d}\right), \quad r \rightarrow 0
$$

where $d=\frac{1}{2 \ell-1}, \mathfrak{D}=\frac{1}{2 d}\left(2 \ell \sin \left(\frac{\pi}{2 \ell}\right)\right)^{-d-1}$ while the values of $\mathcal{C}$ and $\beta$ are now inessential for us.

The behavior of the distribution density $F^{\prime}(r)$ for small $r$ was studied in [Lf2, Theorem 3] in highly general situation. In our case, see also the proof of Theorem $6.2[\mathrm{NN}]$ ) this result of [Lf2] can be rewritten as follows:

$$
F^{\prime}(r) \sim \mathcal{C} \mathfrak{D} d \cdot r^{\beta-d-1} \exp \left(-\mathfrak{D} r^{-d}\right), \quad r \rightarrow 0
$$

This easily means that the asymptotics (3.13) is differentiable w.r.t. $r$.

Substituting (3.14) and (2.12) into (2.10) we obtain

$$
\widetilde{F}(r) \sim \frac{\mathcal{C} \mathfrak{D} d}{\|\varphi\|_{2}} \cdot \sqrt{\frac{2 q}{\pi}} \cdot \int_{0}^{r} \frac{x^{\beta-d-1}}{\sqrt{r-x}} \exp \left(-\mathfrak{D} x^{-d}\right) d x .
$$

\footnotetext{
${ }^{3}$ In [KKW] this equation is written in different but equivalent form.

${ }^{4}$ The case of the operator $L_{X}$ with "separated" boundary conditions was considered earlier in [NN, §7].
} 
Changing the variable $x=r(1-y)$ we get

$$
\widetilde{F}(r) \sim \frac{\mathcal{C} \mathfrak{D} d}{\|\varphi\|_{2}} \cdot \sqrt{\frac{2 q}{\pi}} \cdot r^{\beta-d-\frac{1}{2}} \exp \left(-\mathfrak{D} r^{-d}\right) \int_{0}^{1} \frac{(1-y)^{\beta-d-1}}{\sqrt{y}} \exp \left(-\frac{\mathfrak{D}}{r^{d}}\left((1-y)^{-d}-1\right)\right) d y .
$$

It is easily seen that for $y \geq r^{d / 2}$ the integrand is exponentially small. Therefore, one can integrate only over the interval $\left[0, r^{d / 2}\right]$. In this interval we have $(1-y)^{\beta-d-1} \sim 1$ and $(1-$ $y)^{-d}-1 \sim y d$. Changing the variable $y=r^{d} z$ we arrive at

$$
\begin{aligned}
\widetilde{F}(r) \sim \frac{\mathcal{C} \mathfrak{D} d}{\|\varphi\|_{2}} \cdot \sqrt{\frac{2 q}{\pi}} \cdot r^{\beta-\frac{d+1}{2}} & \exp \left(-\mathfrak{D} r^{-d}\right) \int_{0}^{r^{-d / 2}} \frac{1}{\sqrt{z}} \exp (-d \mathfrak{D} z) d z \sim \\
& \sim \frac{\mathcal{C}}{\|\varphi\|_{2}} \cdot \sqrt{2 q \mathfrak{D} d} \cdot r^{\beta-\frac{d+1}{2}} \exp \left(-\mathfrak{D} r^{-d}\right) \sim \frac{\sqrt{2 q \mathfrak{D} d}}{\|\varphi\|_{2}} \cdot r^{-\frac{d+1}{2}} \cdot F(r) .
\end{aligned}
$$

This gives (3.12).

In the examples considered earlier the small ball behavior of the original processes is well known (the processes $W$ and $B$ are classical ones while the process $\bar{W}_{1}$ was studied in [BNO]). Applying Theorems 1 and 3 we arrive at

Proposition 1. We have, as $\varepsilon \rightarrow 0$,

$$
\begin{aligned}
& \mathbb{P}\left\{\left\|\mathcal{W}_{\mathbf{1}, \alpha}\right\| \leq \varepsilon\right\} \sim\left[\begin{array}{ll}
\frac{4 \varepsilon}{\sqrt{\pi}\left|1-\frac{\alpha}{3}\right|} \cdot \exp \left(-\frac{1}{8} \varepsilon^{-2}\right), & \alpha \neq 3, \\
\frac{2 \varepsilon^{-1}}{\sqrt{3 \pi}} \cdot \exp \left(-\frac{1}{8} \varepsilon^{-2}\right), & \alpha=3
\end{array}\right. \\
& \mathbb{P}\left\{\left\|\mathcal{B}_{1, \alpha}\right\| \leq \varepsilon\right\} \sim\left[\begin{array}{ll}
\frac{2 \sqrt{2}}{\sqrt{\pi}\left|1-\frac{\alpha}{12}\right|} \cdot \exp \left(-\frac{1}{8} \varepsilon^{-2}\right), & \alpha \neq 12, \\
\frac{\varepsilon^{-2}}{\sqrt{6 \pi}} \cdot \exp \left(-\frac{1}{8} \varepsilon^{-2}\right), & \alpha=12
\end{array}\right. \\
& \mathbb{P}\left\{\left\|\mathcal{B}_{\varphi, \alpha}\right\| \leq \varepsilon\right\} \sim\left[\begin{array}{ll}
\frac{2 \sqrt{2}}{\sqrt{\pi}\left|1-\frac{17 \alpha}{5040}\right|} \cdot \exp \left(-\frac{1}{8} \varepsilon^{-2}\right), & \alpha \neq \frac{5040}{17}, \\
\frac{\sqrt{17} \varepsilon^{-2}}{2 \sqrt{21 \pi}} \cdot \exp \left(-\frac{1}{8} \varepsilon^{-2}\right), & \alpha=\frac{5040}{17},
\end{array} \quad \varphi(t)=t(1-t)\right. \\
& \mathbb{P}\left\{\left\|\mathcal{X}_{1, \alpha}\right\| \leq \varepsilon\right\} \sim\left[\begin{array}{ll}
\frac{4 \sqrt{2} \varepsilon^{-\frac{1}{3}}}{\sqrt{3 \pi}\left|1-\frac{\alpha}{120}\right|} \cdot \exp \left(-\frac{3}{8} \varepsilon^{-\frac{2}{3}}\right), & \alpha \neq 120, \\
\frac{\varepsilon^{-\frac{5}{3}}}{3 \sqrt{5 \pi}} \cdot \exp \left(-\frac{3}{8} \varepsilon^{-\frac{2}{3}}\right), & \alpha=120,
\end{array} \quad X(t)=\bar{W}_{1}(t)\right. \\
& \mathbb{P}\left\{\left\|\mathcal{B}_{\varphi, \alpha}\right\| \leq \varepsilon\right\} \quad \sim \quad \frac{2 \sqrt{2}}{\sqrt{\pi}|1-\alpha|} \cdot \exp \left(-\frac{1}{8} \varepsilon^{-2}\right), \quad \alpha \neq 1, \quad \varphi(t)=\frac{1}{\phi\left(\boldsymbol{\Phi}^{-1}(t)\right)}
\end{aligned}
$$

Remark. In the example 5, we cannot apply Theorem 2 (and consequently, also Theorem 3) for $\alpha=1$. 


\section{Some generalizations}

The construction (1.4) can be generalized to a special class of distributions $\varphi$.

Let $\varphi \in D^{\prime}(\mathcal{O})$ satisfy $q \equiv \mathbb{E}|\langle\varphi, X\rangle|^{2}<\infty$. We define a set of Gaussian functions by the formula similar to (1.4):

$$
\mathcal{X}_{\varphi, \alpha}(x)=X(x)-\alpha \psi(x)\langle\varphi, X\rangle, \quad x \in \overline{\mathcal{O}}
$$

where $\psi(x)=\mathbb{E} X(x)\langle\varphi, X\rangle$.

Remark. In terms of the operator theory, the restriction on $\varphi$ means $\varphi \in\left(\operatorname{Im}\left(\mathfrak{G}^{1 / 2}\right)\right)^{\prime}$. This implies $\psi \in \operatorname{Im}\left(\mathfrak{G}^{1 / 2}\right)$, and $q=\langle\varphi, \psi\rangle$. In the random processes theory, $\operatorname{Im}\left(\mathfrak{G}^{1 / 2}\right)$ is called the kernel of distribution for the process $X . \varphi$ is called a linear measurable functional of $X$, see [Lf1, §9].

For the processes (4.1), the Main Lemma and Corollary 1 hold true. Instead of Corollary 2 , we have the following analogue.

Corollary 2'. For $\widehat{\alpha}=\frac{1}{q}$ the process $\mathcal{X}_{\varphi, \widehat{\alpha}}(x)$ and r.v. $\langle\varphi, X\rangle$ are independent. Moreover, $\left\langle\varphi, \mathcal{X}_{\varphi, \widehat{\alpha}}\right\rangle=0$ a.s.

Further, under assumption (2.1) Theorem 1 is valid. If, in addition, the covariance $G_{X}(t, s)$ satisfies the assumptions of $\S 3$, then the algorithm for the construction of KL-expansion for the process $\mathcal{X}_{\varphi, \alpha}$ also runs.

Now we give some examples.

Example 6. Let $X=W$, and let $\varphi(t)=\delta(t-1)$. Then $\psi(t)=G_{W}(t, 1)=t, q=$ $G_{W}(1,1)=1$, and

$$
\mathcal{G}_{\varphi, \alpha}(t, s)=\min \{t, s\}+Q t s \quad\left(Q=\alpha^{2}-2 \alpha\right) .
$$

Thus, for $\alpha \in] 0,2\left[\right.$ the process $\mathcal{W}_{\varphi, \alpha}$ has the same distribution as the Brownian bridge from zero to zero with the length $-\frac{1}{Q}$, see [BoS, 4.4.20].

Theorem 1 gives for $\alpha \neq 1$

$$
\mathbb{P}\left\{\left\|\mathcal{W}_{\varphi, \alpha}\right\| \leq \varepsilon\right\} \sim \frac{4 \varepsilon}{\sqrt{\pi}|1-\alpha|} \cdot \exp \left(-\frac{1}{8} \varepsilon^{-2}\right), \quad \varepsilon \rightarrow 0
$$

This result coincides with a particular case of [Na, Proposition 1.9] and [NP, Theorem 4.1].

Remark. For $\alpha=1$ the process $\mathcal{W}_{\varphi, \alpha}$ has the same distribution as the standard Brownian bridge. It is easy to see that the infinite product in (2.9) diverges, and Theorem 2 is not applicable. The same is true for the forthcoming examples.

Example 7. Let us consider the integrated Wiener process

$$
X(t)=W_{1}^{[0]}(t)=\int_{0}^{t} W(s) d s
$$

$$
\begin{aligned}
& \text { If } \varphi(t)=\delta^{\prime}(t-1) \text { then } \psi(t)=-\left(G_{W_{1}}\right)_{s}(t, 1)=-\frac{t^{2}}{2}, \quad q=\left(G_{W_{1}}\right)_{s t}(1,1)=1, \text { and } \\
& \qquad \mathcal{G}_{\varphi, \alpha}(t, s)=G_{W_{1}}(t, s)+Q \frac{t^{2} s^{2}}{4} \quad\left(Q=\alpha^{2}-2 \alpha\right) .
\end{aligned}
$$


Thus, the process $\mathcal{X}_{\varphi, \alpha}$ has the same distribution as the integrated process from the example 6 . Our Theorem 1 corresponds to the case $m=1$ in [Na, Proposition 1.9]. The small ball behavior in the case $\alpha=1$ (the integrated Brownian bridge) was considered in [Na, Proposition 1.6].

Now let $\varphi(t)=\delta(t-1)$. Then $\psi(t)=G_{W_{1}}(t, 1)=\frac{t^{2}}{2}-\frac{t^{3}}{6}$, and $q=G_{W_{1}}(1,1)=\frac{1}{3}$. Theorem 1 gives for $\alpha \neq 3$

$$
\mathbb{P}\left\{\left\|\mathcal{X}_{\varphi, \alpha}\right\| \leq \varepsilon\right\} \sim \frac{8 \sqrt{6} \varepsilon^{1 / 3}}{\sqrt{\pi}|3-\alpha|} \cdot \exp \left(-\frac{3}{8} \varepsilon^{-2 / 3}\right), \quad \varepsilon \rightarrow 0 .
$$

For $\alpha=3$ the direct calculation shows that $\mathcal{G}_{\varphi, \alpha}(t, s)$ is the Green function of the boundary value problem

$$
u^{I V}=\mu u ; \quad u(0)=u^{\prime}(0)=u(1)=u^{\prime \prime}(1)=0 .
$$

Applying [Na, Theorem 1.4] we obtain

$$
\mathbb{P}\left\{\left\|\mathcal{X}_{\varphi, \alpha}\right\| \leq \varepsilon\right\} \sim \frac{4 \sqrt{2} \varepsilon^{-2 / 3}}{3 \sqrt{\pi}} \cdot \exp \left(-\frac{3}{8} \varepsilon^{-2 / 3}\right), \quad \varepsilon \rightarrow 0
$$

Example 8. Now we consider the Slepian process [S] that is a stationary zero mean-value Gaussian process with the covariance $G_{S}(t, s)=1-|t-s|, t, s \in[0,1]$. Let $\varphi(t)=\delta(t)+\delta(t-1)$. Then $\psi \equiv 1, q=2$, and

$$
\mathcal{G}_{\varphi, \alpha}(t, s)=1+Q-|t-s| \quad\left(Q=2 \alpha^{2}-2 \alpha\right) .
$$

Thus, the process $\mathcal{S}_{\varphi, \alpha}$ has the same distribution as a generalized Slepian process $S^{(c)}, c=1+Q$ (see [GL] and [Na1, §2]). Note that for $Q \geq 0$ also the equality

$$
S^{(c)}(t) \stackrel{d}{=} W(t+c)-W(t), \quad 0 \leq t \leq 1
$$

holds true.

The statement of Theorem 1 corresponds [Na1, Theorem 2.1, part 2]. The small ball behavior in the case $\alpha=1 / 2$ (i.e. $c=1 / 2$ ) is considered in [Na1, Theorem 2.1, part 1].

Example 9. In a similar way one can show that the statement of [Na, Proposition 1.9] for any $m \in \mathbb{N},[\mathrm{Na1}$, Theorem 2.2, part 2] and some theorems of [NP] can be considered as particular cases of our Theorem 1. Thus, this theorem provides a unified approach to many formulas obtained earlier.

One can also consider multiparametric analogues of the transform (1.4). We restrict ourselves to a simplest case.

Let the functions $\varphi_{1}, \varphi_{2} \in L_{1, l o c}(\mathcal{O})$ satisfy the assumption (1.3) and the "orthogonality" assumption 5

$$
\int_{\mathcal{O}} \int_{\mathcal{O}} G_{X}(u, v) \varphi_{1}(u) \varphi_{2}(v) d u d v=0
$$

Let us consider a set of Gaussian functions

$$
\mathcal{X}_{\boldsymbol{\varphi}, \boldsymbol{\alpha}}(x)=X(x)-\alpha_{1} \psi_{1}(x) \int_{\mathcal{O}} X(u) \varphi_{1}(u) d u-\alpha_{2} \psi_{2}(x) \int_{\mathcal{O}} X(u) \varphi_{2}(u) d u, \quad x \in \overline{\mathcal{O}}
$$

\footnotetext{
${ }^{5}$ For the distributions $\varphi_{1}, \varphi_{2} \in\left(\operatorname{Im}\left(\mathfrak{G}^{1 / 2}\right)\right)^{\prime}$ this assumption has the form $\left\langle\varphi_{1}, \mathfrak{G} \varphi_{2}\right\rangle=0$.
} 
where $\psi_{k}=\mathfrak{G} \varphi_{k}, k=1,2$.

It is easy to see that the function (4.2) has the covariance

$$
\mathcal{G}_{\boldsymbol{\varphi}, \boldsymbol{\alpha}}(x, y)=G_{X}(x, y)+Q_{1} \psi_{1}(x) \psi_{1}(y)+Q_{2} \psi_{2}(x) \psi_{2}(y)
$$

where $Q_{k}=q_{k} \alpha_{k}^{2}-2 \alpha_{k}, q_{k}=\left\langle\varphi_{k}, \psi_{k}\right\rangle$. Therefore, one can obtain the $L_{2}$-small ball asymptotics

of the function $\mathcal{X}_{\boldsymbol{\varphi}}, \boldsymbol{\alpha}$ for $\alpha_{k} \neq \frac{1}{q_{k}}, k=1,2$, applying Theorem 1 twice. In the same way we can obtain analogues of other statements.

\section{Appendix}

Lemma 5.1. Let us consider two sequences: $\mu_{k}>0$ and $b_{k} \geq 0, k \in \mathbb{N}$. Let $\sum_{k} \mu_{k}^{-1}<\infty$ and $\sum_{k} b_{k}<\infty$. Then for any $\gamma_{1}, \gamma_{2} \in \mathbb{R}$, as $R \rightarrow \infty$,

$$
\frac{1}{2 \pi} \int_{0}^{2 \pi} \ln \left|\gamma_{1}+\gamma_{2} \sum_{k=1}^{\infty} \frac{b_{k}}{1-\frac{\mu_{k}}{R \exp (i \theta)}}\right| d \theta \longrightarrow \ln \left|\gamma_{1}+\gamma_{2} \sum_{k=1}^{\infty} b_{k}\right|
$$

Proof. Given $\theta \in] 0,2 \pi\left[\right.$, the expression $1-\frac{\mu_{k}}{R \exp (i \theta)}$ is bounded away from zero. By the Lebesgue Dominated Convergence Theorem, we can pass to the limit under the sum sign. Thus, the integrand in (5.1) converges to $\ln \left|\gamma_{1}+\gamma_{2} \sum_{k} b_{k}\right|$ for $\left.\theta \in\right] 0,2 \pi[$, and the convergence is uniform over any segment.

Further, the expression under the absolute value sign has only simple zeros and poles. Therefore, the integrand in (5.1) has only logarithmic singularities, and one can easily construct a summable majorant. Applying again the Lebesgue Theorem, we complete the proof.

I am grateful to Prof. M.A. Lifshits and to Prof. Ya.Yu. Nikitin for stimulating discussions and for some references. Also I am thankful to Prof. M.S. Birman who pointed me out the paper $[\mathrm{Ba}]$.

\section{References}

[AG] N.I. Akhiezer, I.M. Glazman, Theory of linear operators in Hilbert space, 2nd ed.; Moscow, Nauka, 1966 (Russian); English transl. of the 1st ed.: F. Ungar Publ., NY, V.I (1961), V.II (1963).

[Ba] H. Bateman, A formula for the solving function of a certain integral equation of the second kind, Messenger Math., V.37 (1908), 179-187.

[BNO] L. Beghin, Ya. Nikitin, E. Orsingher, Exact small ball constants for some Gaussian processes under the $L_{2}$-norm, ZNS POMI, V.298 (2003), 5-21; J. Math. Sci., V.128 (1995), N1, 2493-2502.

[BS] M.S. Birman, M.Z. Solomyak, Spectral Theory of Self-adjoint Operators in Hilbert Space, Leningrad Univ. Publ., 1980 (Russian); English transl.: Math. and its Applic. Soviet Series, V.5. Kluwer, Dordrecht, 1987. 
[BoS] A.N. Borodin, P. Salminen, Handbook of Brownian Motion: Facts and Formulae, Birkhäuser, Basel, 1996.

[De] P. Deheuvels, A Karhunen - Loève expansion for a mean-centered Brownian bridge, Stat. Prob. Letters, V.77 (2007), N12, 1190-1200.

[DLL] T. Dunker, M.A. Lifshits, W. Linde, Small deviations of sums of independent variables, Progr. Probab. V.43 (1998), 59-74.

[GHT] F. Gao, J. Hannig, F. Torcaso, Comparison theorems for small deviations of random series, Electron. J. Probab. V.8 (2003), N21, 1-17.

[GL] F. Gao, W.V. Li, Small ball probabilities for the Slepian Gaussian fields, Trans. AMS 359 (2007), 1339-1350.

[HN] N. Henze, Ya.Yu. Nikitin, Watson-type goodness-of-fit tests based on the integrated empirical process, Math. Meth. of Statist., V.11 (2002), 183-202.

[KK] L.V. Kantorovich, V.I. Krylov, Approximate methods of higher analysis, 5th ed., Moscow, FML, 1962 (Russian); English transl. of the 3rd ed.: Benster Intersci. Publ., NY, 1958.

[KKW] M. Kac, J. Kiefer, J. Wolfowitz, On tests of normality and other tests of goodness of fit based on the minimum distance method, Ann. Math. Statist., V.26 (1955), N2, 189-211.

[Li] W.V. Li, Comparison results for the lower tail of Gaussian seminorms, J. Theor. Probab. V.5 (1992), N1, 1-31.

[Lf1] M.A. Lifshits, Gaussian Random functions, Kiev, TVMS, 1995 (Russian); English transl.: Math. and its Appl., V.322, Kluwer, Dordrecht, 1995.

[Lf2] M.A. Lifshits, On the lower tail probabilities of some random series, Ann. Prob. V.25 (1997), N1, 424-442.

[Na] A.I. Nazarov, On the sharp constant in the small ball asymptotics of some Gaussian processes under $L_{2}$-norm, Probl. Mat. Anal. V.26 (2003), 179-214 (Russian); English transl.: J. Math. Sci. V.117 (2003), N3, 4185-4210.

[Na1] A.I. Nazarov, Exact $L_{2}$-small ball asymptotics of Gaussian processes and the spectrum of boundary value problems with "non-separated" boundary conditions, http://arxiv.org/abs/0710.1408; to appear in J. of Theor. Prob.

[NN] A.I. Nazarov, Ya.Yu. Nikitin, Exact small ball behavior of integrated Gaussian processes under $L_{2}$-norm and spectral asymptotics of boundary value problems, Probab. Theory and Rel. Fields, V.129 (2004), N4, 469-494.

[NP] A.I. Nazarov, R.S. Pusev, Exact $L_{2}$-small ball asymptotics for some weighted Gaussian processes, SPbMS El. Prepr. Archive, N 2006-1. 16p. (Russian); to appear in ZNS POMI.

[Sl] D. Slepian, First passage time for a particular Gaussian process, Ann. Math. Statist. 32 (1961), 610-612.

[Su] S. Sukhatme, Fredholm determinant of a positive definite kernel of a special type and its application, Ann. Math. Statist., V.43 (1972), N6, 1914-1926. 
[Syt] G.N. Sytaya, On some asymptotic representations of the Gaussian measure in a Hilbert space, Theory of Stochastic Processes, Kiev, V.2 (1974), 93-104 (Russian).

[Ti] E. Titchmarsh, The theory of functions, 2nd ed. Oxford Univ. Press, London, 1939. 